\title{
A versatile and reproducible automatic injection system for liquid standard introduction: application to in-situ calibration
}

\author{
G. Isaacman ${ }^{1}$, N. M. Kreisberg ${ }^{2}$, D. R. Worton ${ }^{1,2}$, S. V. Hering ${ }^{2}$, and A. H. Goldstein ${ }^{1}$ \\ ${ }^{1}$ Department of Environmental Science, Policy, and Management, University of California, Berkeley, California, USA \\ ${ }^{2}$ Aerosol Dynamics Inc., Berkeley, California, USA
}

Received: 31 March 2011 - Published in Atmos. Meas. Tech. Discuss.: 25 May 2011

Revised: 22 August 2011 - Accepted: 5 September 2011 - Published: 19 September 2011

\begin{abstract}
The quantitation of trace organic compounds in ambient organic aerosol is difficult due to the chemical complexity of these mixtures, but is needed to provide insight into their sources and formation processes. Compound-level characterization of organic aerosols is typically performed through sample collection followed by gas or liquid chromatography. With these methods, introduction of liquid standards has long been used as an effective means of quantifying trace compounds, but automating this technique for use with in-situ instrumentation has not previously been achieved. Here we develop an automatic injection system (AutoInject) for the introduction of liquids into a custom collection and analysis cell for improved quantitation in chromatographic measurements. The system consists of chilled reservoirs containing liquid standards from which a sample loop is loaded and then injected into the cell. The AutoInject is shown to be reproducible over 106 injections with a relative standard deviation of $1.5 \%$, and have negligible injection-to-injection carryover. A 6-port selector allows injection of different liquid standards separately or simultaneously. Additionally, automatic injection of multiple sample loops is shown to generate a linear multi-point calibration curve. Tests conducted in this work focus on use with the Thermal desorption Aerosol Gas chromatograph (TAG), but the flexibility of the system allows it to be used for a variety of applications.
\end{abstract}

\section{Introduction}

Ambient atmospheric aerosol is known to contain thousands of organic compounds in trace amounts (Goldstein and Galbally, 2007), making accurate characterization of aerosol composition difficult. However, a detailed quantitative

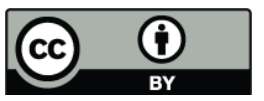

Correspondence to: G. Isaacman (gabriel.isaacman@berkeley.edu) understanding of this composition has significant benefits; speciation of organic aerosol improves source attribution by quantifying specific tracers known to originate from a given source. These tracers include directly emitted compounds, such as levoglucosan from woodburning and hopanes from diesel exhaust, as well as secondary compounds formed through the oxidation of volatile organic compounds (VOCs), such as phthalic acid and furanones (Rogge, 1993; Schauer et al., 1996; Fraser and Lakshmanan, 2000; Mazurek, 2002; Simoneit, 2005; Williams et al., 2010). Detailed analysis of aerosol composition has also contributed to a better understanding of formation mechanisms and atmospheric oxidation processes (Gao et al., 2004; Jaoui et al., 2008; Surratt et al., 2008; Winterhalter et al., 2009). Consequently, a number of methods have been developed to speciate aerosol and quantify useful tracers. Most of this work has relied on filter collection and subsequent chromatographic separation, either using thermal desorption gas chromatography (TD-GC) or solvent extraction followed by liquid chromatography (LC) or GC.

Accurate quantitation using chromatographic methods relies on measuring detector response relative to directly introduced authentic standards using a multi-point calibration, often with a correction for variation in sample recovery or detector drift. The most common technique for correcting for run-to-run variability is to spike each filter with a known quantity of some liquid internal standard (Mazurek et al., 1989; Schauer et al., 1996; Offenberg et al., 2011). However, though this method has been used with great success to quantify compounds on filters, it poses a significant drawback when applied to in-situ data collection; specifically, the 24-h per day presence and vigilance of an operator. An alternate method appropriate to field measurements is the use of a regularly injected complex mixture - a "tracking standard" - to monitor long-term trends in detector response, coupled with regular multi-point calibrations (Kreisberg et al., 2009). Though shown to be effective, this field method assumes

Published by Copernicus Publications on behalf of the European Geosciences Union. 
monotonic changes in detector response and does not correct for variations related to changes in transfer efficiency and detector response. Therefore, combining these two methods by injecting both internal standards on every run as well as regular tracking standards would greatly improve the accuracy of in-situ quantitative speciation. This dual calibration approach is the objective of the automatic injection system (AutoInject) described in this work. The AutoInject was developed and tested specifically for the Thermal desorption Aerosol Gas chromatograph (TAG) field instrument, an insitu quantitative gas chromatograph used to study aerosol composition (see Williams et al., 2006 for details).

Common laboratory-based liquid introduction systems typically do not meet requirements for field use. The most common mechanisms in laboratory data collection are "XYZ" automatic systems, in which a robotic arm with a syringe withdraws an aliquot from a vial containing a known liquid, moves to the instrument inlet, and injects the syringe (e.g. those sold by Agilent Technologies for GC or Waters Technologies for LC). This approach is infeasible in the case of custom field instruments, however, as (a) it usually requires vertical injection which is not possible on some instruments (including TAG), (b) frequent septum piercing caused by syringe injection leads to coring and sample contamination, and (c) it is typically bulky, involves many moving parts, and usually does not provide temperature stabilization.

Several field-applicable standard introduction systems have been developed for high pressure liquid chromatography (HPLC) and gas-phase calibration. Automated liquid introduction systems have been recently developed for LC (van Midwoud et al., 2011) in which flow rate is controlled by syringe pumps. These systems have the capability to switch between liquids by controlling pump rates and valve states, but the volumes of solvent used (in some cases many milliliters per injection) generates more waste than is preferred for field use. Jardine et al. (2010) recently developed an elegant system that uses a stepper motor to introduce liquid into a helium stream at much lower flow rates to be evaporated and delivered to a TD-GC or other in-situ instrument. However, due to the low vapor pressure of many compounds relevant to atmospheric aerosols, such a scheme is impractical for the study of particle-phase compounds.

In this work we develop a new method for the introduction of fixed volumes of liquid into a custom cell that is versatile and quantitative. Field deployment is emphasized by generating minimal waste (less than $30 \mu \mathrm{l}$ per injection) and relying on pressure gradients to create flow, thereby minimizing moving parts. Several hundred injections were tested to address the ability of the automatic injection system to meet the following goals:

1. Injection of a known volume must be reproducible.

2. Injection-to-injection carryover must be minimal and the presence of the system must have a negligible effect on blanks.
3. Quantity of liquid should be variable to facilitate multipoint calibrations.

4. Manual injection should be possible, allowing for injection of standards not contained within the system.

\section{AutoInject description}

A schematic of the Automatic Injection System (AutoInject), which occupies a total space of $40 \mathrm{~cm} \times 35 \mathrm{~cm} \times 15 \mathrm{~cm}$, is shown in Fig. 1a. Pressurized reservoirs, selected using a multiport selector, deliver liquid to fill a sample loop of known volume, which is injected via a 6-port valve into a custom cell. Up to four reservoirs of liquid are kept in a custom refrigerator consisting of an insulated, machined aluminum block cooled using an on-off controlled Peltier device (Custom Thermoelectric) capable of sustaining sub-freezing temperatures. The reservoirs are pressurized to $30 \mathrm{psig}$ with helium distributed through a 6-way manifold. Reservoirs (shown in Fig. 1b) consist of a standard $10 \mathrm{~mm} \times 75 \mathrm{~mm}$ Pyrex test tube capped with a $10 \mathrm{~mm}$ compression fitting (Swagelok Company) using teflon ferrules. The cap has two drilled holes, through which $1 / 16$ in outer diameter (o.d.) stainless steel tubing is inserted and silver brazed, with one tube reaching to the bottom of the reservoir for liquid delivery and one tube at the top for pressurization. The liquid delivery tube of each reservoir is connected to a 6-port selector valve (Upchurch Scientific V-1241) by $50 \mathrm{~cm}$ of $125 \mu \mathrm{m}$ diameter (i.d.), 1/16 in o.d. tubing made of polyether ether ketone (PEEK), a non-reactive, opaque polymer, which prevents exposure to light. The small i.d. of this tubing minimizes the volume of liquid that is temporarily stored outside of the cooled block and is therefore not cooled. The 6-port selector is connected by $20 \mathrm{~cm}$ of $60 \mu \mathrm{m}$ i.d. PEEK tubing to a 6-port valve (Rheodyne MXP7900) configured for sample loop injection. All liquid downstream of the selector must be injected or discarded during each injection cycle, so small i.d. tubing is necessary to minimize waste. All tubing was purchased from Upchurch Scientific.

\subsection{Operation procedure}

With the 6-port valve in the load position (pictured in Fig. 1), the liquid loads a sample loop of known volume (see Sect. 2.2), with excess going to a waste container; in this case, waste is injected onto glass wool at the bottom of a vial containing activated charcoal, facilitating evaporation of solvent that is then scrubbed to prevent emissions. In the inject position, the sample loop is flushed with helium into the custom cell via a $15 \mathrm{~cm} \times 1 / 32$ in o.d. $\times 250 \mu \mathrm{m}$ i.d. stainless steel capillary. Injection helium is restricted by $1 \mathrm{~m}$ of $60 \mu \mathrm{m}$ tubing, maintaining helium flow below $2 \mathrm{ccm}$. Additionally, a check valve on this line prevents back flow from the cell even in the presence of a pressure gradient. Following injection, excess liquid remaining in the lines is purged 

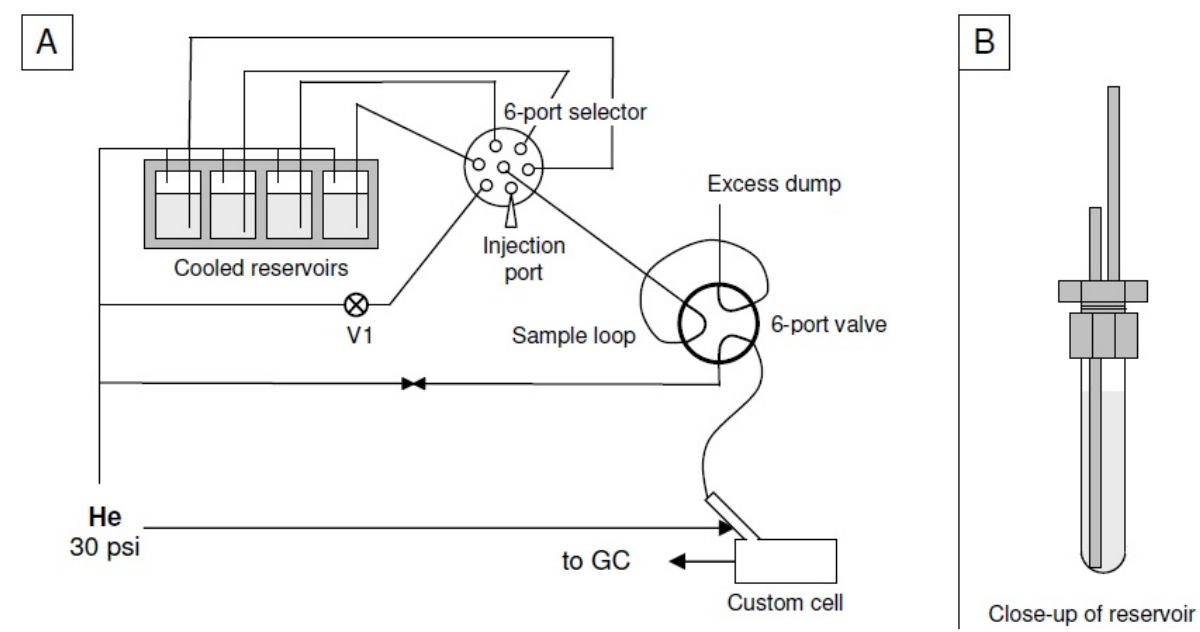

Fig. 1. Overview of the automatic injection system (AutoInject) showing (a) the schematic diagram of the system in "load" and (b) a close-up of a standard reservoir. The custom cell used in these studies is the collection and thermal desorption (CTD) cell of the TAG instrument (Williams et al., 2006).

with helium through the selector port connected directly to helium. When idle, the helium port is selected with the shut-off valve (V1) closed, so neither liquid nor helium is wasted and the restriction of the injection helium minimizes helium waste. All valves (6-port selector, 6-port valve, and helium valve) are actuated automatically through computer controlled contact closures using a custom written Labview program (National Instruments Corporation).

The custom cell into which liquid was introduced for this study is the collection and thermal desorption (CTD) cell on the Thermal desorption Aerosol Gas chromatograph (TAG) described in Kreisberg et al. (2009). This CTD cell has a built-in helium purged injection port with a septum seal for needle insertion for manual injections. The injection capillary of the AutoInject was inserted into this port with a compression fitting (Swagelok Company), eliminating the need for the septum. The injected liquid standard was thermally desorbed at $300^{\circ} \mathrm{C}$ and analyzed using a gas chromatograph (GC) coupled for most tests to a flame ionization detector (FID; Agilent Technologies) and for carryover tests to a time-of-flight mass spectrometer (Kronus ToF-MS; Scientific Analysis Instruments).

A typical injection cycle lasts approximately 45 seconds per reservoir used: approximately $20 \mathrm{~s}$ to load and inject a single loop volume and $15 \mathrm{~s}$ to helium purge the line between the valve and selector, with some time for transitions in between. For all experiments in this study, injection of a standard was followed by injection of solvent to clean the lines. Minimizing loop load time minimizes waste and load timing is strongly dependent on helium pressure and tubing i.d., so the shortest load time possible to fully load the loop was determined.

\subsection{Sample loop}

The sample loop used in these tests was a $15 \mathrm{~cm} \times 1 / 16$ in o.d. $\times 180 \mu \mathrm{m}$ i.d. stainless steel sample loop (Rheodyne). The internal volume injected, consisting of the loop itself plus dead-volume within the valve and fittings, was found to be $4.0 \pm 0.1 \mu \mathrm{l}$ for the conditions and valve described above by comparing FID response of one loop of standard to a multi-point calibration using the same standard done by manual injection. Any 1/16 in o.d. tubing can be used as a sample loop, making the AutoInject highly flexible for custom applications.

\section{Results}

\subsection{Stability tests}

The two primary necessities for a useful automatic injection system are the capability for long-term, highly reproducible injections, and the capability to switch standards with minimal cross contamination.

The reproducibility of liquid injections using the AutoInject was studied through the injection of a standard con-

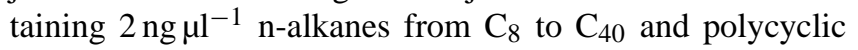
aromatic hydrocarbons (PAHs) spanning a similar volatility range in chloroform. All tests focus on the volatility range of $\mathrm{C}_{15}$ to $\mathrm{C}_{35}$ as this is where nearly all compounds are observed by TAG. In Fig. 2, the FID response to compounds in each run, relative to the average response across 106 injections of each compound, demonstrates the high reproducibility of the AutoInject. The dashed line marks the standard deviation, $\sigma$, of $1.5 \%$. The root mean square (RMS) error for all peaks is below $2.5 \%$ and averages $1.4 \%$. Some compounds that partially co-elute in the chromatographic separation have been 


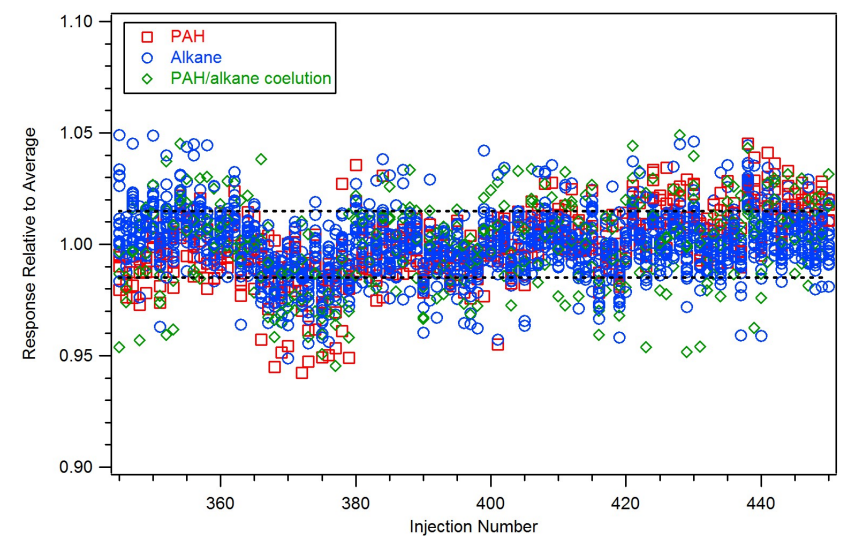

Fig. 2. Stability of the AutoInject over 106 runs. FID detector response of a given compound is shown relative to the average response of that compound over all runs shown. Symbols differentiate PAHs, alkanes, and co-eluting peaks that contain both PAHs and alkanes while the dashed line represents the standard deviation of relative response for all standard compounds measured $(1.5 \%)$.

binned in order to minimize measuring errors not intrinsic to the AutoInject, especially difficulties in drawing baselines for, and therefore accurately quantifying, co-eluting peaks. However, deviations from unity are, in most cases, not well correlated between different compounds $\left(R^{2}=0.27 \pm 0.16\right)$, suggesting that a large fraction of error is not from AutoInject instability (i.e. injecting more or less liquid) but rather from other sources, such as inaccuracies in chromatogram integration due to chromatographic baseline variations.

In continuous operation, a reservoir, which has a volume of approximately $3 \mathrm{ml}$, contains enough liquid for more than 150 injections under the conditions and timing used in these tests. In the case of the TAG instrument, this allows the instrument to be run for at least one week, with hourly internal standard injections, without the need to refill a reservoir or expose the operator to chemicals. This length of time is of course dependent on the volume of the sample loop, the volume of the transfer lines, the volume of the reservoirs, and the duration of the load timing.

\subsection{Carryover tests}

The liquid carryover from one injection to the next was quantified by alternating between injection of a standard containing a complex 110-component mixture representing classes known to be atmospherically relevant (n-alkanes, PAHs, aromatic acids, straight chain acids, ketones, esters, etc.) and injection of an equal volume of a similar complex mixture containing 21 deuterated compounds. The number of solvent rinses following each injection was varied to determine the volume of solvent injection necessary to eliminate carryover. To accurately quantify carryover, a ToF-MS was used, allowing for specific ion extraction and, consequently, an improved level of detection. While the absence

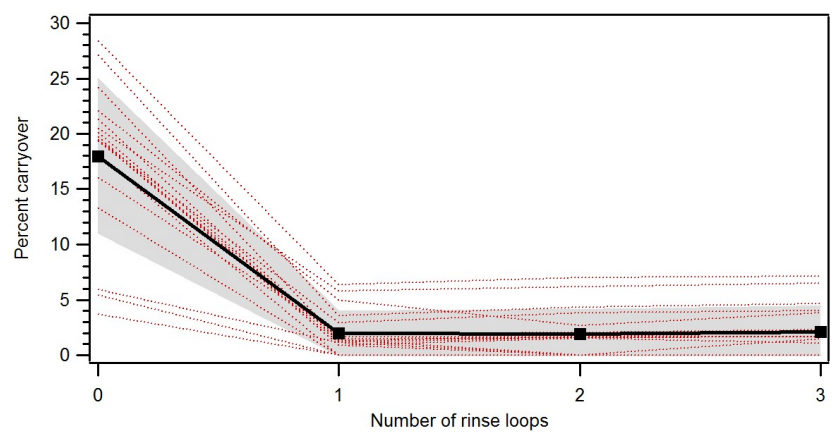

Fig. 3. Injection-to-injection carryover tests for 18 representative compounds (dashed red lines) and overall average (solid black line with standard deviation in gray area). Compounds shown are a selection of deuterated alkanes (n-hexadecane- $d_{34}, n$-eicosane- $d_{42}$, n-tetracosane- $\mathrm{d}_{50}, \mathrm{n}$-octacosane- $\mathrm{d}_{58}, \mathrm{n}$-dotriacontane- $\left.\mathrm{d}_{66}\right)$, deuterated polycyclic aromatic hydrocarbons (dPAHs; phenanthrene- $\mathrm{d}_{10}$, fluoranthene- $\mathrm{d}_{10}$ ), straight chain acids (hexadecanoic acid, octadecanoic acid, eicosanoic acid), PAHs (phenanthrene, fluoranthene), and other atmospheric relevant compounds ( $5 \alpha$-cholestane- $\mathrm{d}_{6}, 5 \alpha$ cholestane, 2-pentadecanone, retene, benzophenone, and dihydro5-octyl-2(3H)-furanone).

of a solvent rinse results in significant carryover (sometimes greater than $20 \%$ ), this is greatly reduced by a single solvent rinse (Fig. 3). No statistically significant decrease in carryover was observed when employing more than a single rinse, suggesting that carryover after a single solvent rinse is negligible and any remaining carryover is external to the AutoInject.

Given low carryover, injection of standards from two different reservoirs in the same injection cycle is possible. Injection of PAHs and alkanes in the same injection cycle from two different reservoirs was shown to yield the same FID response within reproducibility error as injection of each separately.

\subsection{Multi-point calibration tests}

Useful capabilities beyond the necessary requirements of the AutoInject were also tested, including injection of multiple loops of a standard, and manually loading the sample loop.

Multi-point calibrations were performed by injection of one to three loops of $2 \mathrm{ng} \mathrm{\mu l}^{-1} \mathrm{n}$-alkanes in chloroform followed by one loop of solvent (Fig. 4). The order of multiloop injections within a "calibration cycle" was varied (1-23, 3-1-2, 2-3-1, etc.) with an injection of a single loop of standard in between each calibration cycle (the calibration cycles are shown connected by lines in Fig. 4). In each of the two series of calibration cycles (red squares and blue circles in Fig. 4), detector response is shown relative to the average response of single loop injections that were interspersed between the calibration cycles. Solvent blanks show the carryover to be less than $1 \%$ in these series. These calibrations lie on to the 1:1 line, as expected, with an overall slope of 


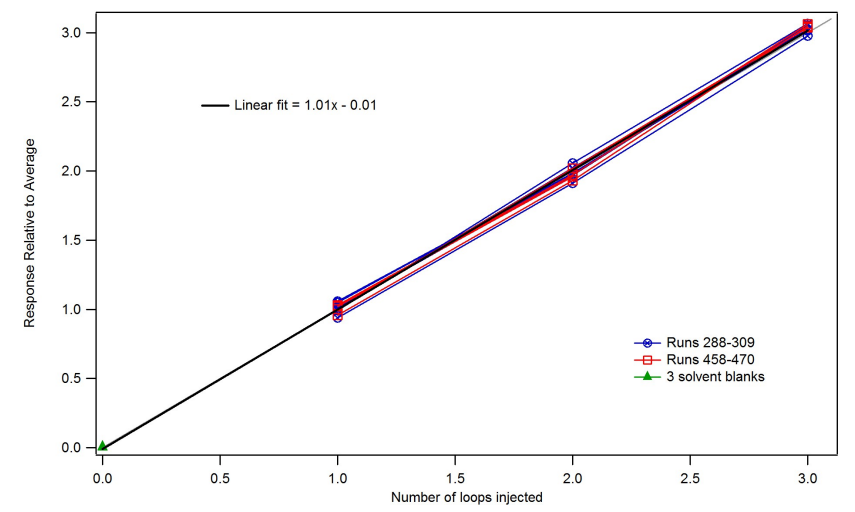

Fig. 4. Demonstration of injection of multiple loops of straight chain alkanes $\left(\mathrm{C}_{15}\right.$ to $\left.\mathrm{C}_{35}\right)$ used as a multi-point calibration. Connected points are "calibration cycles" performed at two different times in the testing (blue and red, see Sect. 3.2 for further discussion). Average FID detector response is shown relative to the average response for single loops injections interspersed between these calibrations. A linear fit (black line) is on top of the 1:1 line (gray line).

$1.01 \pm 0.01$ and an intercept of $-0.01 \pm 0.02$, demonstrating the capability of using the AutoInject for generating multipoint calibration curves.

\subsection{Manual loading tests}

A manual injection port (Rheodyne) connected to one port of the 6-port selector (shown in Fig. 1) was used to manually fill the sample loop. This "pseudo-manual" injection method allows for injection of small amounts of any liquid without needing to fill a reservoir and showed similar reproducibility $(\sigma=2.3 \%)$ to automated injections. This capability extends the use of multi-point calibration methods by allowing for the occasional injection of significantly more concentrated or dilute standards than are necessary for automated running conditions (i.e. weekly pseudo-manual injections of a very dilute standard) without the need for an additional reservoir.

\section{Conclusions}

In this work, a new device and method for the reproducible injection of liquid standards, dubbed the "AutoInject", was developed. Multiple chilled reservoirs were connected to a $4.0 \pm 0.1 \mu \mathrm{l}$ sample loop via a 6-port selector (used to select a reservoir) and a 6-port-valve (used to load and inject the sample loop). Liquid injection with the AutoInject was shown to be highly reproducible, with a relative standard deviation of $1.5 \%$ and negligible carryover after one solvent rinse. Use of the 6-port selector allows for up to three different liquid standards plus a solvent rinse reservoir to be available for injection, either alternated or simultaneously. Each reservoir contains enough liquid for more than 150 injections.
Furthermore, through injection of multiple loops, the AutoInject was shown to generate linear multi-point calibrations. The range of these calibrations can be extended beyond the range of standards in the reservoirs through the use of an injection port to manually load the sample loop.

Through these capabilities, the AutoInject is able to fulfill a variety of liquid injection needs. For instance, internal standards can be injected onto every sample, while a tracking standard can be used for regular calibration and/or multi-point calibrations. Multi-reservoir switching capabilities greatly extend the utility of the system, allowing for use of multiple standards in each run, such as co-injection of internal and calibration standards to be used for cross calibration or regular injection of two standards that cannot be stored together. Regular introduction of standards will greatly improve the quantitation of instruments such as the TAG and other instrumentation measuring a similar range of chemicals. Furthermore, waste generation is kept low in this system, minimizing ecological impact and personal hazard of calibration in the field.

The components of the AutoInject make it a highly versatile device that can be adapted to any instrument that relies on regular liquid injections for any purpose. Injection volume is variable through sample loop selection, loop load times are dependent on reservoir pressure, and reservoir volumes can be increased by using larger test tubes. The flexibility of the injection capillary allows for injection into any custom sample cell. In addition, the AutoInject is ideal for field use as it is compact, versatile, and relies on a minimum of moving parts for increased reliability.

In future studies using the TAG instrument, the AutoInject will be used for repeated injections of an internal standard, as well as regular multi-point calibrations and tracking standards. This system could also be used for regular injection of a derivatizing agent, greatly expanding the capabilities of insitu gas chromatography. The combined capabilities of this automated calibration system offers the potential to significantly improve the accuracy and extend the utility of TAG and other particle-phase in-situ instrumentation.

Acknowledgements. Many thanks to A. Lambe, B. Williams, and T. Hohaus for field-testing a prototype system. This work was supported by the US Department of Energy STTR program, DE-FG02-05ER-86235 and DE-FG02-08ER-86335, and the National Science Foundation Atmospheric Chemistry Program Grants 0922562 and 0931934 . Gabriel Isaacman was funded by the US Environmental Protection Agency (EPA) Science to Achieve Results (STAR) program, Fellowship Assistance Agreement no. FP-91781901-0. This work has not been formally reviewed by EPA. The views expressed in this work are solely those of the authors, and EPA does not endorse any products or commercial services mentioned.

Edited by: E. C. Apel 


\section{References}

Fraser, M. P. and Lakshmanan, K.: Using Levoglucosan as a Molecular Marker for the Long-Range Transport of Biomass Combustion Aerosols, Environ. Sci. Technol., 34, 4560-4564, 2000.

Gao, S., Keywood, M., Ng, N. L., Surratt, J., Varutbangkul, V., Bahreini, R., Flagan, R. C., and Seinfeld, J. H.: LowMolecular-Weight and Oligomeric Components in Secondary Organic Aerosol from the Ozonolysis of Cycloalkenes and $\alpha$ Pinene, J. Phys. Chem. A, 108, 10147-10164, 2004.

Goldstein, A. H. and Galbally, I.: Known and unexplored organic constituents in the earth's atmosphere, Environ. Sci. Technol., 41, 1514-1521, 2007.

Jaoui, M., Edney, E. O., Kleindienst, T. E., Lewandowski, M., Offenberg, J. H., Surratt, J. D., and Seinfeld, J. H.: Formation of secondary organic aerosol from irradiated a-pinene/toluene/ $/ \mathrm{NO}_{\mathrm{x}}$ mixtures and the effect of isoprene and sulfur dioxide, J. Geophys. Res., 113, D09303, doi:10.1029/2007JD009426, 2008.

Jardine, K. J., Henderson, W. M., Huxman, T. E., and Abrell, L.: Dynamic Solution Injection: a new method for preparing pptvppbv standard atmospheres of volatile organic compounds, Atmos. Meas. Tech., 3, 1569-1576, doi:10.5194/amt-3-15692010, 2010.

Kreisberg, N. M., Hering, S. V., Williams, B. J., Worton, D. R., and Goldstein, A. H.: Quantification of Hourly Speciated Organic Compounds in Atmospheric Aerosols, Measured by an InSitu Thermal Desorption Aerosol Gas Chromatograph (TAG), Aerosol Sci. Tech., 41, 38-52, 2009.

Mazurek, M. A.: Molecular Identification of Organic Compounds in Atmospheric Complex Mixtures and Relationship to Atmospheric Chemistry and Sources, Environ. Health Persp., 110, 995-1003, 2002.

Mazurek, M. A., Cass, G. R., and Simoneit, B. R.: Interpretation of High-Resolution Gas Chromatography and High-Resolution Gas Chromatography/Mass Spectrometry Data Acquired from Atmospheric Organic Aerosol Samples, Aerosol Sci. Tech., 10, 408420, 1989.
Offenberg, J. H., Lewandowski, M., Jaoui, M., and Kleindienst, T. E.: Contributions of Biogenic and Anthropogenic Hydrocarbons to Secondary Organic Aerosol during 2006 in Research Triangle Park, NC, Aerosol Air Qual. Res., 99-108, 2011.

Rogge, W. F.: Molecular Tracers for Sources of Atmospheric Carbon Particles: Measurements and Model Predictions, Ph. D. thesis, California Institute of Technology, 1993.

Schauer, J. J., Rogge, W. F., Hildemann, L. M., Mazurek, M. A., and Cass, G. R.: Source apportionment of airborne particulate matter using organic compounds as tracers, Atmos. Environ., 30, 3837-3855, 1996.

Simoneit, B. R.: A review of current applications of mass spectrometry for biomarker/molecular tracer elucidation, Mass Spectrom. Rev., 24, 719-765, 2005.

Surratt, J. D., Go, Y., Chan, A. W., Vermeylen, R., Shahgholi, M., Kleindienst, T. E., Edney, E. O., Offenberg, J. H., Lewandowski, M., Jaoui, M., Maenhaut, W., Claeys, M., Flagan, R. C., and Seinfeld, J. H.: Organosulfate Formation in Biogenic Secondary Organic Aerosol, J. Phys. Chem. A, 112, 8345-8378, 2008.

van Midwoud, P. M., Janssen, J., Merema, M. T., Graaf, I. A., Groothuis, G. M., and Verpoorte, E.: On-line HPLC Analysis System for Metabolism and Inhibition Studies in Precision-Cut Liver Slices, Anal. Chem., 83, 84-91, 2011.

Williams, B. J., Goldstein, A. H., Kreisberg, N. M., and Hering, S. V.: An In-Situ Instrument for Speciated Organic Composition of Atmospheric Aerosols: Thermal Desorption Aerosol GC/MSFID (TAG), Aerosol Sci. Tech., 40, 627-638, 2006.

Williams, B. J., Goldstein, A. H., Kreisberg, N. M., Hering, S. V., Worsnop, D. R., Ulbrich, I. M., Docherty, K. S., and Jimenez, J. L.: Major components of atmospheric organic aerosol in southern California as determined by hourly measurements of source marker compounds, Atmos. Chem. Phys., 10, 11577-11603, doi:10.5194/acp-10-11577-2010, 2010.

Winterhalter, R., Herrmann, F., Kanawati, B., Nguyen, T. L., Peeters, J., Vereecken, L., and Moortgat, G. K.: The gas-phase ozonolysis of beta-caryophyllene $(\mathrm{C}(15) \mathrm{H}(24))$, Part I: an experimental study, Phys. Chem. Chem. Phys., 11, 4152-4172, 2009. 